\title{
LNG-solid impacts with gas cushioning and phase change
}

Peter D. Hicks*

School of Engineering, Fraser Noble Building, King's College, University of Aberdeen, Aberdeen, AB24 3UE, UK

\begin{abstract}
LNG-solid impacts with gas cushioning and phase change are investigated theoretically in a coupled inviscid liquid and viscous gas regime. Condensation from the gas to the LNG is driven by local increases in the gas pressure above the saturation vapour pressure. This is modelled as a sink term on the kinematic boundary condition at the gas interface. To leading order, the bulk liquid motion is unaffected by condensation, with its evolution governed by the same boundary integral equation used in models of non-volatile gas-cushioned liquid-solid impacts. The proposed model extends the approach used to describe two-dimensional non-volatile gas-cushioned impacts by incorporating phase change and is applied to a range of physically relevant LNGsolid impacts associated with sloshing. As an LNG free-surface approaches touchdown with a solid wall, a gas pressure build-up occurs in the gap separating liquid from solid, which decelerates and deforms the liquid freesurface. This deformation of the free surface may result in gas entrapment. In non-volatile impacts, pockets of trapped gas are associated with oscillatory pressure signals, while previous experiments have shown that these oscillations may be damped by phase change in impacts involving volatile liquids. Compared to impacts with non-volatile liquids, gas condensation is shown to reduce both the impact pressures and the volume of gas trapped. Depending on the impact parameters, the proposed model differentiates between cases where a pocket of trapped gas may or may not be formed. A criterion on the critical normal impact velocity above which gas entrapment is not expected is obtained. This indicates that across a range of length scales that are physically relevant to LNG sloshing, gas entrapment is not expected for impact velocities greater than $0.05 \mathrm{~m} \mathrm{~s}^{-1}$. Impacts where gas compressibility is important are investigated, as well as impacts into corners of containment tanks with varying angles. The model developed is suitable for the analysis of small and medium-sized LNG-solid impacts, as well as larger-scale sloshing model tests involving impacts of water cushioned by water vapour. The importance of inertia in the gas is identified in larger scale impacts.
\end{abstract}

Keywords: LNG, sloshing, liquid-solid impact, gas cushioning, phase change

${ }^{*}$ Corresponding author
Email address: p.hicks@abdn.ac.uk (Peter D. Hicks) 


\section{Introduction}

The transport of liquefied natural gas (LNG) by ship occurs at atmospheric pressure, when the LNG is close to thermodynamic equilibrium with its gas phase. The resulting storage temperature of $-163^{\circ} \mathrm{C}$ poses many challenges for the structural containment of LNG, as the walls of the containment tank are constructed out of plywood [6], to improve the thermal insulation of the tank. Sloshing of LNG induced by global ship motions produces violent impacts between the LNG and the tank walls. During an inspection of the $138,000 \mathrm{~m}^{3}$ capacity LNG carrier Spirit of Catalunya in 2006, damage to the containment tank was observed [16]. This has motivated further analysis of LNG sloshing impacts, to understand the induced loads and reduce the risk of a loss of containment.

The behaviour of LNG during sloshing has been investigated, both experimentally and theoretically, to accurately predict the pressures and loads on the containment tank walls [see e.g. 12, 15]. A feature of many liquid-solid impacts, including those associated with liquid sloshing, is the entrapment and entrainment of gas. This occurs due to interactions between the liquid, the wall and the gas separating the liquid from the wall prior to impact. Such phenomena occur across a wide range of impact length scales and momenta, ranging from sea wave impacts with coastal defences [30], to impacts of individual droplets on solid substrates [22]. Pockets of trapped gas or regions of aerated liquid, which expand and contract during impact, give rise to oscillatory pressures signals $[36,3,4]$. Oscillatory pressures have been measured experimentally in a pocket of gas trapped in the corner of a tank by a free-surface wave [2] and in wave impacts with vertical walls [25]. In wave impacts with vertical walls, flip-through events that result in vertical jets running up the wall, may or may not involve gas entrainment [23]. Three different flip-through modes have been identified, which are referred to as: mode (a) flip-through events without gas entrainment, mode (b) flip-through events with a single well define gas cavity and mode (c) flip-through events involving a region of small scale gas and liquid mixing [24, 25]. The importance of gas entrainment to sloshing has led to further numerical investigation of this phenomena using smooth particle hydrodynamics [11, 10, 39, 17], and boundary element methods [1].

A sketch of a gas pocket trapped in the corner of a tank is shown in figure 1, alongside other flows associated with LNG sloshing that involve gas entrapment and entrainment. Ordered by an increasing typical characteristic length scale, these include (a) a droplet of LNG approaching a wall, (b) a jet of LNG running up the tank wall and approaching a corner of the tank, (c) a free surface wave hitting the roof of the tank, (d) the tip of a breaking wave as it approaches impact with a wall and (e) a pocket of gas trapped in the corner of a tank. In each case the approximate initial region of impact cushioning by the gas is bounded by a dashed line. The flow associated with the impact of an individual droplet is not usually considered when investigating LNG sloshing, as the pressure induced by droplet impacts are not appreciable in the context of the forces and loads associated with larger sloshing impacts. However, droplet impact cushioning is briefly described herein to highlight the analogies that exist with existing gas cushioning models, which are more 
frequently applied to smaller scale phenomena.
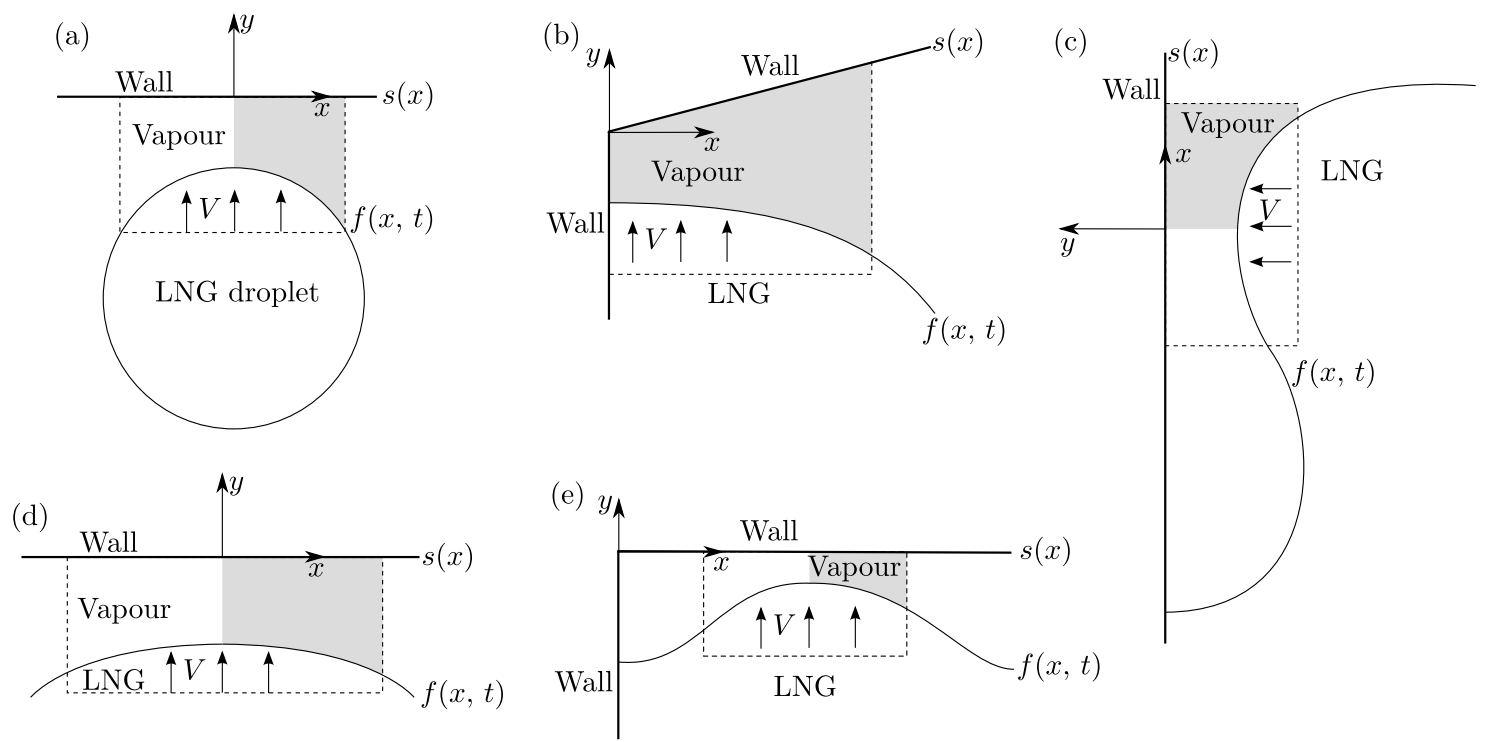

Figure 1: A sketch of different types of gas-cushioned liquid-solid impacts associated with LNG sloshing, presented in approximate order of increasing size, showing (a) an LNG droplet approaching the roof of a tank, (b) a tip of a liquid jet running up the side of a tank wall and approaching a corner of a tank, (c) a tip of a breaking wave as it approaches impact with a wall, (d) LNG rising up to hit the roof of a tank and (e) LNG trapping a gas pocket in the corner of a tank. In each case the region enclosing the initial vapour cushioning is bounded by a dotted line, while the grey shaded areas indicate the spatial domain illustrated in subsequent plots of the free-surface evolution.

The pressure and temperature associated with LNG storage conditions and the closeness of thermodynamic equilibrium, indicate that phase change is likely to occur in LNG impacts. Except for Leidenfrost droplet impacts [see e.g. 33], phase change is not commonly associated with other liquid-solid impact applications. However, it can have a marked impact on LNG impacts, where experiments have shown that phase change may damp the pressure oscillations associated with non-volatile gas cushioning [26, 41]. This damping is due to pressure increases driving vapour condensation, while pressure decreases result in LNG vaporisation. Hence rather than periodically compressing and expanding a fixed mass of trapped gas during an impact, the gas can change phase. If condensation reduces the volume of a gas cavity formed in wave impacts with a vertical wall, then following the classification of [23], the occurrence of mode (a) flip-through events (which do not involve entrained gas), is expected to increase, while mode (b) and (c) events (which involve the gas phase), will be less prevalent.

Unlike the two-dimensional model presented herein, theoretical analyses of LNG-solid impacts with phase change have previously been restricted to extensions of the one-dimensional piston model of breaking wave impact of Bagnold [7]. Extensions of the piston model, due to Braeunig et al. [8] and Ancellin et al. [5], include phase change between gas and liquid, and predict the damping of the pressure signal observed in 
experiments. However, in these models the local impact structure and flow behaviour is lost. In the current paper, the phase change in gas-cushioned LNG impacts will be investigated by extending the viscous gas and inviscid liquid impact cushioning model of Smith et al. [35]. Recently, this distinguished limit has more often been used to model smaller scale gas-cushioned droplet impacts [27, 19]. However, Smith et al. [35] envisages the use of this model in larger scale impacts. The coupled viscous gas with inviscid liquid limit differs from the earlier impact cushioning model of Wilson [37], who looked at inviscid gas cushioning for liquid-solid impacts with more inertia than those considered here. In both the viscous and inviscid gas cushioning models, as liquid approaches touchdown with a solid surface, a pressure build-up in the gas region separating the liquid from the solid wall is predicted. This increased pressure decelerates the liquid, delaying the instant of initial touchdown beyond the time predicted in the absence of cushioning. The deceleration and subsequent deformation of the liquid free-surface results in the formation of a trapped gas pocket, as the free surface close to the initial point of minimum separation is overtaken by the surrounding free surface.

For non-volatile cushioning, the viscous gas and inviscid liquid limit gives rise to scaling laws, which predict the horizontal and characteristic vertical extent of the trapped gas pocket. Excellent agreement with these scaling laws has been obtained in both drop tests [20] and droplet impacts [22]. The model of Smith et al. [35] has been extended to three spatial dimensions [19], and to include surface tension [32, 27, 13], gas compressibility [20], and rarefied gas effects [14].

The extension of the viscous gas and inviscid liquid cushioning model to incorporate phase change will be accomplished by drawing on ideas associated with the evaporation from, and condensation into thin liquid films [see e.g. 9, 28, 38]. In the context of volatile LNG impact cushioning, this results in a mass flux from the vapour film into the liquid, induced by a local vapour pressure in excess of the saturation vapour pressure. The details of the incorporation of phase change in the viscous gas and inviscid liquid cushioning model are described in $\S 2$. Section 3 uses the resulting model to investigate flow configurations in sloshing that are associated with gas cushioning, while $\S 4$ contains conclusions and further discussion.

\section{Model development}

The LNG and gas cushion are both assumed to behave as isothermal fluids, with a common temperature of $\tilde{T}=-161.48^{\circ} \mathrm{C}$, which is the saturation temperature of methane at standard atmospheric pressure. LNG is a mixture of hydrocarbons and other trace elements, with composition varying in different oil and gas

fields. However, roughly $90 \%$ of LNG is methane, and consequently, unless otherwise stated, fluid properties of pure methane are used. The gas phase is assumed to behave like an ideal gas, with the gas pressure $\tilde{p}_{g}$, and density $\tilde{\rho}_{g}$, related by

$$
\tilde{p}_{g}=\tilde{\rho}_{g} \mathcal{R}_{g} \tilde{T} .
$$


Here the specific gas constant of pure methane $\mathcal{R}_{g}=518.3 \mathrm{~J} \mathrm{~kg}^{-1} \mathrm{~K}^{-1}$. During storage and transport, the gas phase may also include compounds other than methane. However, for simplicity, cushioning of liquid methane is investigated in a pure methane gas environment. Previous studies of gas compressibility have predicted gas temperature increases due to adiabatic vapour compression [27] and viscous dissipation [20]. However, these temperature increases are typically quite small and will consequently be neglected.

In common with most studies of violent liquid impact, body forces due to gravity are neglected in the momentum balance, as the characteristic Froude number for such flows is large. In this case the mass and momentum conservation for a compressible Newtonian fluid are governed by the Navier-Stokes equations

$$
\begin{aligned}
\frac{\partial \tilde{\rho}_{g}}{\partial \tilde{t}}+\tilde{\nabla} \cdot\left(\tilde{\rho}_{g} \tilde{\boldsymbol{u}}_{g}\right) & =0, \\
\tilde{\rho}_{g}\left(\frac{\partial \tilde{\boldsymbol{u}}_{g}}{\partial \tilde{t}}+\tilde{\boldsymbol{u}}_{g} \cdot \tilde{\nabla} \tilde{\boldsymbol{u}}_{g}\right) & =-\tilde{\nabla} \tilde{p}_{g}+\mu_{g} \tilde{\nabla}^{2} \tilde{\boldsymbol{u}}_{g}+\left(\mu_{g}+\lambda_{g}\right) \tilde{\nabla}\left(\tilde{\nabla} \cdot \tilde{\boldsymbol{u}}_{g}\right) .
\end{aligned}
$$

Here the velocity $\tilde{\boldsymbol{u}}=(\tilde{u}, \tilde{v})$ in directions $\tilde{x}$ parallel to a flat wall and $\tilde{y}$ normal to the wall and pointing away from the liquid (see figure 1). The fluid viscosities, which are assumed to be constant, are denoted $\mu_{g}$ and $\lambda_{g}$, respectively. The time $\tilde{t}$ is chosen such that in the absence of pre-impact cushioning, the initial touchdown occurs at $\tilde{t}=0$. In these equations, a subscript $g$ denotes a property of the gas phase. Similar equations, with a subscript $l$ denoting a liquid property, govern mass and momentum conservation in the liquid, except here liquid compressibility is neglected.

At the liquid-gas interface, the kinematic boundary condition reads

$$
\tilde{\jmath}=\tilde{\rho}_{g}\left(\tilde{\boldsymbol{u}}_{g}-\tilde{\boldsymbol{u}}_{i}\right) \cdot \hat{\boldsymbol{n}}=\tilde{\rho}_{l}\left(\tilde{\boldsymbol{u}}_{l}-\tilde{\boldsymbol{u}}_{i}\right) \cdot \hat{\boldsymbol{n}}
$$

while the normal stress balance implies

$$
\tilde{\jmath}\left(\frac{1}{\rho_{l}}-\frac{1}{\tilde{\rho}_{g}}\right)+\hat{\boldsymbol{n}} \cdot\left(\mathbb{T}_{g}-\mathbb{T}_{l}\right) \cdot \hat{\boldsymbol{n}}=\gamma \tilde{\nabla} \cdot \hat{\boldsymbol{n}},
$$

and the tangential stress balance implies

$$
\hat{\boldsymbol{n}} \cdot\left(\mathbb{T}_{g}-\mathbb{T}_{l}\right) \cdot \hat{\boldsymbol{t}}=0
$$

Here we have assumed Marangoni effects on the free surface are negligible, while

$$
\hat{\boldsymbol{n}}=\left(-\frac{\partial \tilde{f}}{\partial \tilde{x}}, 1\right) / \sqrt{1+\left(\frac{\partial \tilde{f}}{\partial \tilde{x}}\right)^{2}} \text { and } \hat{\boldsymbol{t}}=\left(1, \frac{\partial \tilde{f}}{\partial \tilde{x}}\right) / \sqrt{1+\left(\frac{\partial \tilde{f}}{\partial \tilde{x}}\right)^{2}}
$$

are a unit normal vector (pointing into the gas), and a unit normal tangential vector to the liquid freesurface $\tilde{y}=\tilde{f}(\tilde{x}, \tilde{t})$. These expressions relate the mass flux due to condensation $\tilde{\jmath}$, to the interfacial velocity $\tilde{\boldsymbol{u}}_{i}$, the gas stress tensor $\mathbb{T}_{g}$, the liquid stress tensor $\mathbb{T}_{l}$, and the surface tension $\gamma$. In the normal stress balance, it is assumed that terms corresponding to vapour recoil and also to the work done by both compression and viscous dissipation can be neglected. 
The condensation mass flux

$$
\tilde{\jmath}=\frac{1}{\sqrt{2 \pi \mathcal{R}_{g}}}\left(\sigma_{g} \frac{\tilde{p}_{g}}{\sqrt{\tilde{T}_{g}}}-\sigma_{l} \frac{\tilde{p}_{e}\left(\tilde{T}_{l}\right)}{\sqrt{\tilde{T}_{l}}}\right)
$$

where $\tilde{p}_{e}\left(\tilde{T}_{l}\right)$ is the saturation vapour pressure, and $\sigma_{g}$ and $\sigma_{l}$ are the accommodation constants for the gas and liquid phases [29]. Appropriate values of $\sigma_{g}$ and $\sigma_{l}$ are still debated [see 31]. However, in the following we assume they are equal and take the value one. Non-unity accommodation constants can be readily considered through modifications of the non-dimensional parameter values. Equations of this type are often used when modelling evaporation and condensation from a thin liquid layer coating a flat surface, when the saturation vapour pressure is linearized for small variations in temperature [9, 28]. However, if the flow is isothermal, then both the gas and liquid temperatures equal the constant temperature $\tilde{T}$, and hence the saturation vapour pressure $\tilde{p}_{e}(\tilde{T})$ is also constant. In this case condensation is driven by the local gas pressure induced by cushioning $\tilde{p}_{g}$, exceeding the vapour saturation pressure $\tilde{p}_{e}(\tilde{T})$. This differs from classical analyses of evaporation from a thin liquid film, where (for one-sided models), the ambient gas pressure is constant, with evaporation induced by a heat flux through the liquid, which raises the surface temperature of the liquid film.

\subsection{Non-dimensionalization and model simplification}

For an impact with characteristic normal liquid velocity $V$ and length scale $R$, which is chosen to be the radius of curvature of the liquid free-surface close to the point of initial touchdown, non-dimensional lengths and velocities in the liquid are defined to be

$$
\left(\tilde{x}, \tilde{y}, \tilde{u}_{l}, \tilde{v}_{l}\right)=\left(\varepsilon R x, \varepsilon R y, V u_{l}, V v_{l}\right)
$$

while in the gas these quantities and additionally a non-dimensional gas density is defined to be

$$
\left(\tilde{x}, \tilde{y}, \tilde{u}_{g}, \tilde{v}_{g}, \tilde{\rho}_{g}\right)=\left(\varepsilon R x, \varepsilon^{2} R y, \frac{V}{\varepsilon} u_{g}, V v_{g},\left[\rho_{g}\right] \rho_{g}\right) .
$$

Here $\varepsilon$ is a small parameter, whose value will be determined subsequently. This small parameter is used to focus the analysis on a small region of liquid (of size $\varepsilon R$ by $\varepsilon R$ ), and a small region of gas (of horizontal size $\varepsilon R$ and vertical size $\varepsilon^{2} R$ size), close to the point of touchdown. It is expected that the gas pressure build-up is only strong enough to cushion and deform the liquid free-surface in these coupled regions. Cushioning behaviour occurs when the vertical height of the gas layer is much smaller than the horizontal extent of the cushioning region, as gas confinement is required to generate the necessary pressure build-up. Consequently, the characteristic vertical height of the gas layer is smaller (by a factor of $\varepsilon$ ), than its horizontal extent. The fluid velocities are all scaled by the characteristic impact velocity $V$, except for the horizontal gas velocity, which is faster (by a factor of $\varepsilon^{-1}$ ), to conserve the mass of gas in the small aspect ratio gas layer. The 
characteristic scale for the gas density $\left[\rho_{g}\right]=\tilde{p}_{e}(\tilde{T}) / \mathcal{R}_{g} \tilde{T}$, is determined from the ambient pressure and temperature.

Common to both the liquid and the gas; a non-dimensional time, free-surface position, solid-body position, pressure and condensation flux are defined, satisfying

$$
(\tilde{t}, \tilde{f}, \tilde{s}, \tilde{p}, \tilde{\jmath})=\left(\frac{\varepsilon^{2} R}{V} t, \varepsilon^{2} R f, \varepsilon^{2} R s, \tilde{p}_{e}(\tilde{T})+\frac{\rho_{l} V^{2}}{\varepsilon} p,\left[\rho_{g}\right] V j\right)
$$

The characteristic time scale is the time it takes for the liquid free-surface (with characteristic velocity $V$ ), to traverse the $\varepsilon^{2} R$ height of the gas layer in the absence of cushioning. The scaling of the free-surface height and the body position naturally match the vertical scaling of the gas layer, as these two interfaces mark the vertical boundaries of the gas. A characteristic pressure scale $\rho_{l} V^{2} / \varepsilon$, is employed in both the liquid and gas to retain the pressure in the liquid momentum equation. A common pressure scale in both liquid and gas is necessary for cushioning, as the liquid evolution is unaffected by a gas pressure smaller than this. The characteristic scale for the condensation mass flux $\left[\rho_{g}\right] V$, is motivated by a balance between $\tilde{\jmath}$ and the difference between the gas and interfacial velocities in the kinematic boundary condition (3).

For $\rho_{l} /\left[\rho_{g}\right] \gg 1$ and $\varepsilon \ll 1$, the leading-order kinematic boundary condition for the liquid is

$$
v_{l}=\frac{\partial f}{\partial t} \quad \text { on } \quad y=0
$$

while the corresponding leading-order kinematic boundary condition for the gas is

$$
v_{g}=\frac{\partial f}{\partial t}+\frac{j}{\rho_{g}} \quad \text { on } \quad y=f(x, t)
$$

Here the disparate horizontal and vertical gas velocity scales resolve to give no-slip conditions for the gas on both the gas-liquid and gas-solid interfaces. To leading order, the boundary conditions on the liquid are identical to the non-volatile case, with phase change only entering the problem through the boundary condition on the gas. The condensation mass flux (7) becomes

$$
j=C p_{g},
$$

where the reciprocal of the condensation number

$$
C=\frac{\rho_{l} V}{\varepsilon\left[\rho_{g}\right] \sqrt{2 \pi \mathcal{R}_{g} \tilde{T}}} .
$$

The size of $\varepsilon$ is determined by balancing the pressure gradient with the largest viscous term in the horizontal component of the momentum equation in the gas $(2 \mathrm{~b})$, which gives

$$
\varepsilon=\left(\frac{\mu_{g}}{\rho_{l} V R}\right)^{1 / 3} .
$$


If a parameter $I=\left[\rho_{g}\right] /\left(\varepsilon \rho_{l}\right)$, measuring the importance of inertia in the gas film is defined, then inertia in the gas is negligible for $I \ll 1$. Additionally, if $\varepsilon \ll 1$, then from equation (2), the leading-order behaviour of the gas is given by

$$
\frac{\partial \rho_{g}}{\partial t}+\frac{\partial}{\partial x}\left(\rho_{g} u_{g}\right)+\frac{\partial}{\partial y}\left(\rho_{g} v_{g}\right)=0, \quad-\frac{\partial p_{g}}{\partial x}+\frac{\partial^{2} u_{g}}{\partial y^{2}}=0, \quad \text { and } \quad \frac{\partial p_{g}}{\partial y}=0 .
$$

Here the final equation, corresponding to vertical momentum conservation, implies $p_{g}=p_{g}(x, t)$, so that the gas pressure is constant across the gas film. Upon employing no-slip conditions on the boundaries of the gas film, the horizontal velocity in the gas film

$$
u_{g}=\frac{1}{2} \frac{\partial p_{g}}{\partial x}(y-s)(y-f)
$$

The ideal gas equation implies

$$
\rho_{g}=1+\kappa p_{g}
$$

where the non-dimensional parameter $\kappa=\rho_{l} V^{2} /\left(\varepsilon \tilde{p}_{e}(\tilde{T})\right)$, measures the ratio of the dynamic pressure induced in the gas by the liquid motion, to the ambient gas pressure. If $\kappa \ll 1$, then the induced pressure is much smaller than the ambient gas pressure, and hence to leading order the gas can be considered incompressible. Conversely for $\kappa=\mathcal{O}(1)$, the pressure induced in the gas by the liquid motion is equal to the ambient gas pressure, and hence gas compressibility is integral to the leading-order cushioning behaviour. The condition $\kappa \ll 1$, gives rise to a condition on the characteristic impact velocity [27]:

$$
V \ll\left(\frac{\tilde{p}_{e}(\tilde{T})^{3} \mu_{g}}{\rho_{l}^{4} R}\right)^{1 / 7},
$$

for impacts in which the gas may be assumed to be incompressible.

If $\varepsilon \ll 1$ and the liquid Reynolds number $R e_{l}=\rho_{l} V R / \mu_{l} \gg 1$, then the leading-order liquid behaviour is governed by the linearized Euler equations

$$
\frac{\partial u_{l}}{\partial x}+\frac{\partial v_{l}}{\partial y}=0, \quad \frac{\partial u_{l}}{\partial t}=-\frac{\partial p_{l}}{\partial x}, \quad \text { and } \quad \frac{\partial v_{l}}{\partial t}=-\frac{\partial p_{l}}{\partial y} .
$$

Given the boundary condition (11) on the liquid free-surface, the free-surface acceleration can be related to the pressure gradient along the free surface, through [37, 35]

$$
\frac{\partial^{2} f}{\partial t^{2}}=\frac{1}{\pi} f_{-\infty}^{\infty} \frac{p_{l, \xi} \mathrm{d} \xi}{\xi-x}
$$

At leading order, the normal stress boundary condition (4), connects the pressure in the liquid to the pressure in the gas at the free surface, through

$$
p_{l}-p_{g}=\Gamma \frac{\partial^{2} f}{\partial x^{2}}
$$

where the non-dimensional parameter $\Gamma=\varepsilon \gamma /\left(\rho_{l} V^{2} R\right)$, which is the reciprocal of the reduced Weber number. This quantity measures the importance of surface tension relative to inertia in the LNG. 


\subsection{Parameter investigation and coupled model equations}

(a) LNG

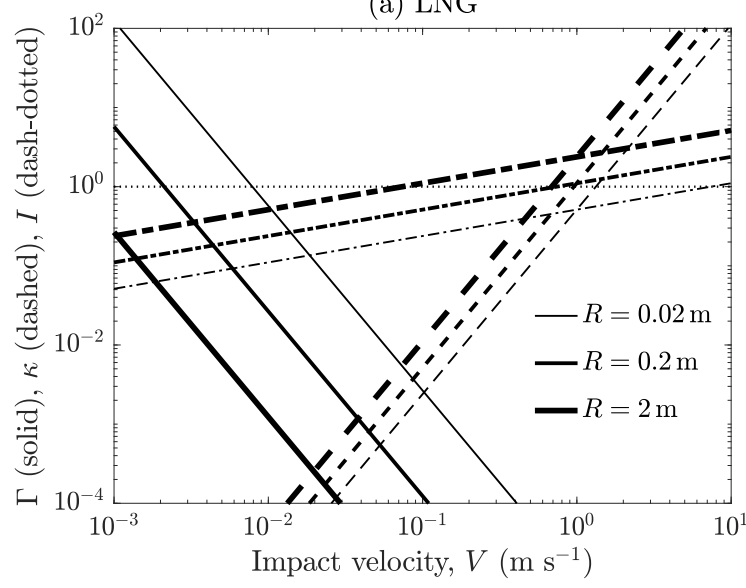

(b) Water-water vapour

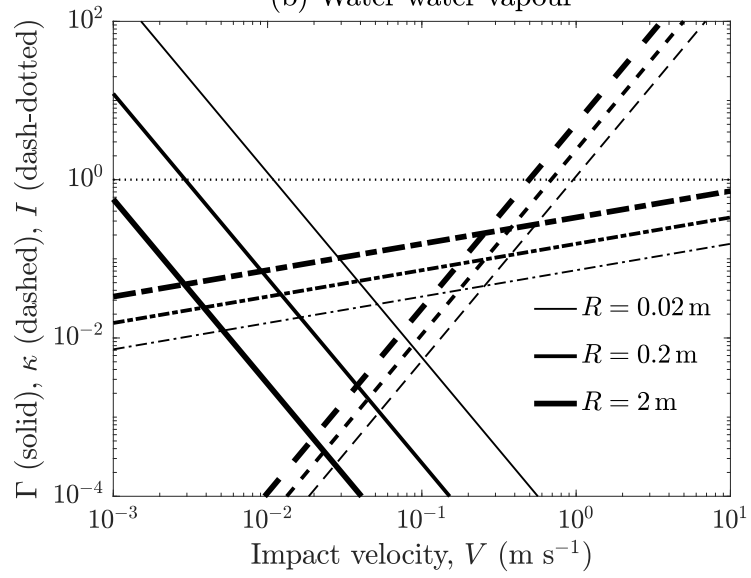

(c) Water-air

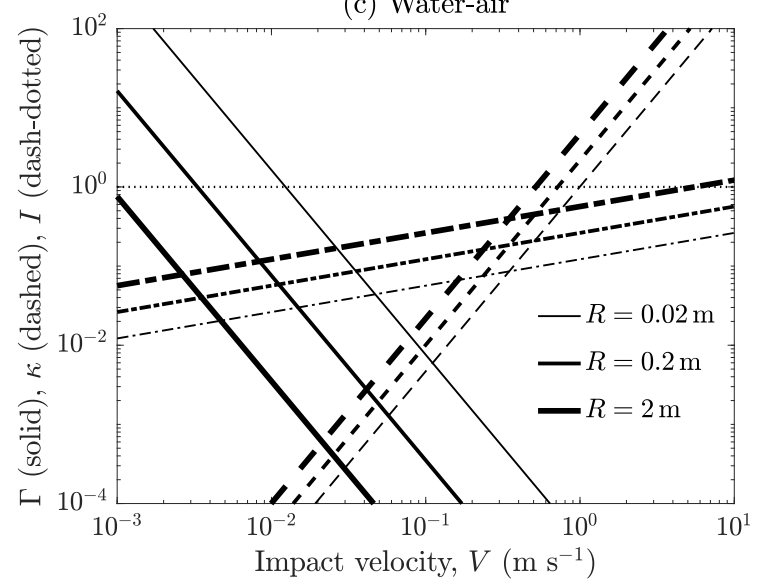

Figure 2: The magnitude of the surface tension parameter $\Gamma$ (solid line), the compressibility parameter $\kappa$ (dashed line) and the gas inertia parameter $I$ (dash-dotted line), as a function of impact velocity $V$, for impact length scales $R=2 \mathrm{~m}$, $0.2 \mathrm{~m}$ and $0.02 \mathrm{~m}$. Values of the impact parameters are calculated for (a) an LNG-vapour impact at $-161.48^{\circ} \mathrm{C}$, (b) a water-water vapour impact at $100^{\circ} \mathrm{C}$ and (c) a water-air impact at $25^{\circ} \mathrm{C}$.

To assess how the model parameters for LNG vapour-cushioned LNG impacts and also for the water vapour-cushioned water impacts of [26] differ from the corresponding model parameters associated with the more widely studied problem of air-cushioned water impacts, figure 2 shows the values of $\Gamma, \kappa$ and $I$ as functions of the characteristic normal impact velocity $V$ for (a) LNG impacts at $-161.48^{\circ} \mathrm{C}$, (b) water-water vapour impacts at $100^{\circ} \mathrm{C}$ and (c) water-air impacts at $25^{\circ} \mathrm{C}$. Each parameter is calculated for characteristic impact length scales $R=2 \mathrm{~m}$ (thick line), $0.2 \mathrm{~m}$ and $0.02 \mathrm{~m}$ (thin line). The difference in the values of the parameters are due to variations in the fluid densities, viscosities and surface tensions. At standard atmospheric pressure, the saturation temperature for pure methane $\tilde{T}=-161.48^{\circ} \mathrm{C}$, while the ambient ideal 
Table 1: Non-dimensional parameter values for $V=0.05 \mathrm{~m} \mathrm{~s}^{-1}$ and $R=0.05 \mathrm{~m}$. To calculate $C$ for water-air impacts, we assume the phases are volatile and are in thermodynamic equilibrium.

\begin{tabular}{|c|ccc|}
\hline Parameter & LNG & Water-water vapour & Water-air \\
\hline$R e_{l}$ & 8950 & 2800 & 8480 \\
$\varepsilon$ & 0.0161 & 0.0194 & 0.0171 \\
$\Gamma$ & 0.0039 & 0.0112 & 0.0084 \\
$\kappa$ & 0.00064 & 0.0013 & 0.0014 \\
$I$ & 0.256 & 0.0609 & 0.0359 \\
$C$ & 1.238 & 2.945 & 4.572 \\
\hline
\end{tabular}

gas density $\tilde{\rho}_{g}=1.75 \mathrm{~kg} \mathrm{~m}^{-3}$, the LNG density $\rho_{l}=422.4 \mathrm{~kg} \mathrm{~m}^{-3}$, the gas viscosity $\mu_{g}=4.46 \times 10^{-6} \mathrm{Pas}$ and the surface tension coefficient $\gamma=0.013 \mathrm{~N} \mathrm{~m}^{-1}$. For impacts with characteristic normal velocity $V=$ $0.05 \mathrm{~m} \mathrm{~s}^{-1}$ and characteristic length scale $R=0.05 \mathrm{~m}$, the corresponding values of $R e_{l}$ and $\varepsilon$ are given in table 1 , alongside the values of the other non-dimensional parameters. The values $R e_{l}$ and $\varepsilon$ justify neglecting viscous terms in the liquid and terms of size $\mathcal{O}(\varepsilon)$, in each of the three different impact types.

For any given $V$ and $R$, the values of $\Gamma$ and $\kappa$ are slightly smaller in an LNG impact than in both the equivalent water-water vapour and water-air impacts, indicating that surface tension and gas compressibility are less important in LNG impacts. To investigate the influence of phase change and gas compressibility in impact cushioning, terms in the model involving $C$ and $\kappa$ will be retained, while terms involving surface tension will be neglected. Gas compressibility is retained while surface tension is neglected, because gas compressibility becomes more important at higher impact velocities, while surface tension is less important (see figure 2).

The value of the gas inertia parameter $I$ in an LNG impact significantly exceeds the corresponding value in either a water-water vapour or a water-air impact, and hence gas inertia is more important in LNG impacts. This is primarily because the gas-liquid density ratio of methane at $-161.48^{\circ} \mathrm{C}$ is larger than both the water-water vapour density ratio at $100^{\circ} \mathrm{C}$ and the water-air density ratio at $25^{\circ} \mathrm{C}$. For $V=0.05 \mathrm{~m} \mathrm{~s}^{-1}$ and $R=0.05 \mathrm{~m}$, the values of $I$ are given in table 1 for each impact type. For LNG impacts with $R=0.05 \mathrm{~m}$ and $V=0.05 \mathrm{~m} \mathrm{~s}^{-1}$; the gas inertia parameter $I=0.256$, and hence the assumption that $I \ll 1$ is only valid for LNG impacts with smaller $V$ or smaller $R$. For both water-water vapour or water-air impacts, the assumption that $I \ll 1$ is satisfied over a much larger range of $R$ and $V$. The water-vapour cushioned scale model tests of [26] and [41] were conducted in a tank of length $1.25 \mathrm{~m}$, breadth $0.1 \mathrm{~m}$ and height $0.4 \mathrm{~m}$, while characteristic impact velocities of $1.6 \mathrm{~m} \mathrm{~s}^{-1}$ were reported. For $R$ less than the tank length, the effect of gas inertia is negligible in impacts of the size of the [26] and [40] scale model tests. Consequently, for impacts up to this size, the full range of flow configurations shown in figure 1 may be investigated with the current 
theory.

For LNG impacts, the modelling assumption that $I \ll 1$, means that impacts of LNG droplets and impacts of LNG jets running up tank walls into tank corners (figure 1(a-b)), may be investigated. However, only small breaking wave impacts, LNG impacts with the tank roof and LNG impacts trapping gas in the corner of the tank (figure 1(c-e)), may be studied without further developing the theory to incorporate inertia in the LNG vapour. Moderate inertia in thin fluid films may be incorporated by extending the theory of Sivapuratharasu et al. [34], while extensions of the inviscid gas cushioning model of Wilson [37] may be appropriate in even higher momentum impacts. In both cases, the inherent extendibility of our phase change analysis would allow condensation to be incorporated in higher gas inertia LNG impacts. Our current aim is to understand the flow physics and effects of phase change, and consequently, only impacts satisfying $I \ll 1$ will be considered, to isolate the effects of phase change from gas inertia.

Integrating the gas mass conservation equation (16a) from the gas-liquid interface $y=f(x, t)$ to the solid wall $y=s(x)$, and using equation (13) to eliminate the evaporative mass flux, gives rise to a modified Reynolds lubrication equation of the form

$$
\frac{\partial}{\partial t}\left[\left(1+\kappa p_{g}\right)(s-f)\right]-C p_{g}=\frac{1}{12} \frac{\partial}{\partial x}\left[\left(1+\kappa p_{g}\right)(s-f)^{3} \frac{\partial p_{g}}{\partial x}\right] .
$$

For $\kappa>0$ and $f(x, t)<s(x)$, this gives a parabolic equation with which to update the gas pressure. For $\Gamma=0$, the normal stress balance (22) implies the liquid and gas pressure are equal across the interface, and hence the free-surface position and gas pressure are additionally coupled using equation (21). The evolution of this coupled system of equations, from an initial free-surface profile of $f(x, t)=-\frac{1}{2} x^{2}-t$ and gas pressure of $p_{g}(x, t)=0$ at large negative times, is computed numerically using the method outlined in Hicks \& Purvis [21]. Throughout the impact $f(x, t) \sim-\frac{1}{2} x^{2}-t$ and $p_{g}(x, t) \rightarrow 0$ as $|x| \rightarrow 0$. While a range of initial undisturbed free-surface shapes could be chosen, the particular function of $f(x, t)$ used for large negative times, is consistent with an initially circular free surface, which is then scaled with the disparate horizontal and vertical length scales in the gas, to approximate locally the LNG interface.

\section{Results and discussion}

\subsection{Variation in condensation number $C$}

Figure 3 illustrates the evolution of the liquid free-surface (top) and pressure (bottom), for (a) $C=0$, (b) $C=2$ and (c) $C=4$. Profiles corresponding to the grey shaded areas in figure 1 are shown for $\kappa=0$, indicating gas compressibility is neglected, in addition to gas inertia and surface tension, while the wall position $s(x)=0$. In (a), the non-volatile case $(C=0)$, there is a pressure build-up in the gas layer as the liquid free-surface moves upwards towards the tank wall. This pressure build-up causes the liquid freesurface to decelerate. This deceleration of the free surface is initially greatest where the separation between 
(a) $C=0$
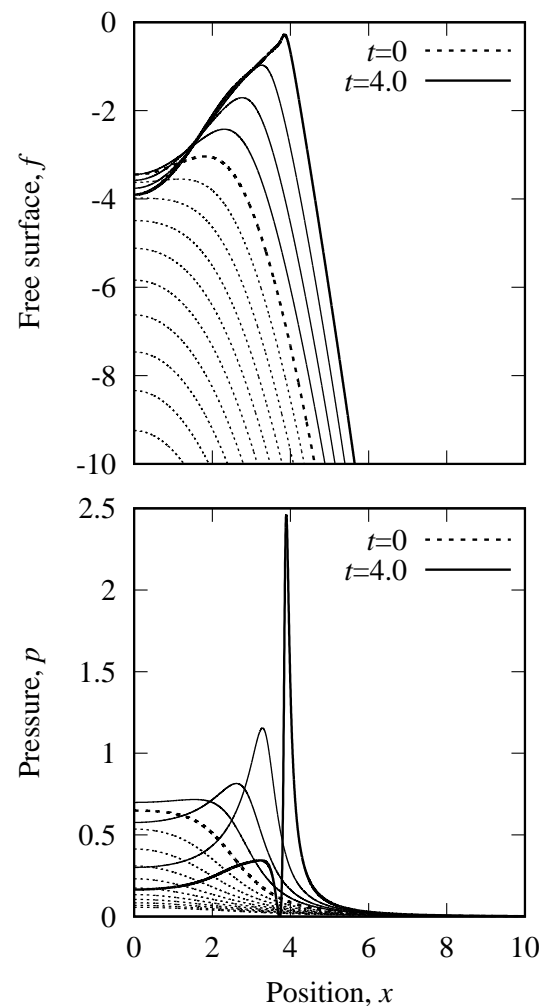

(b) $C=2$
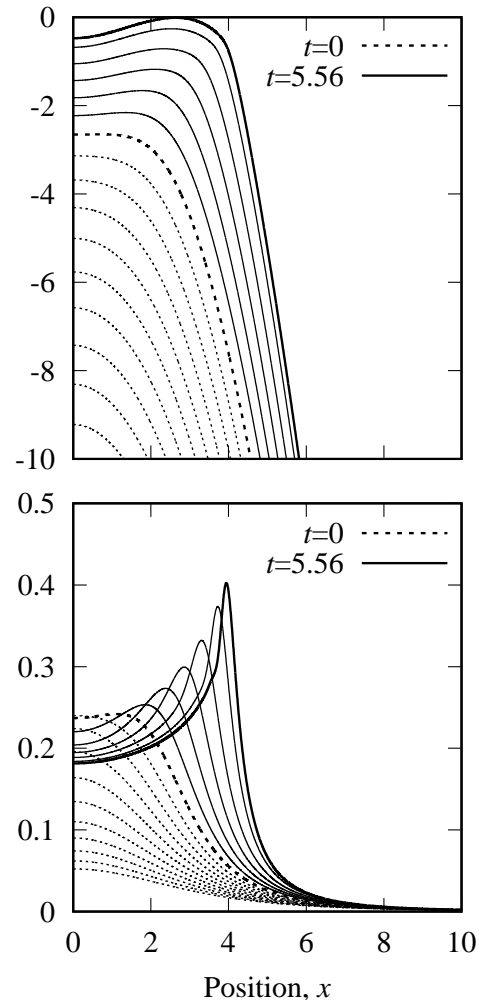

(c) $C=4$
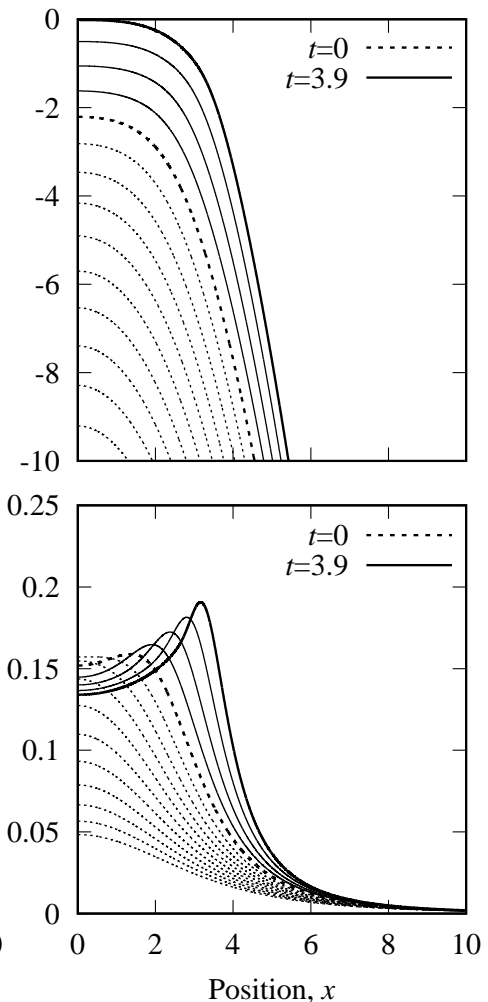

Figure 3: Profiles of the free surface (top) and pressure (bottom) for $\kappa=0$ and $s(x)=0$, with (a) $C=0$, (b) $C=2$, and (c) $C=4$. Profiles are shown at integer non-dimensional time increments, except for the final profile, which is shown just before touchdown. Profiles are symmetric about the vertical axis, with the solution domain matching the grey shaded regions of figure 1. The horizontal Note that the pressure profiles are shown with different vertical scales to illustrate their features.

the liquid and wall is least, i.e. at $x=0$. Eventually the surrounding free-surface, which is decelerated less, overtakes the free surface at $x=0$, trapping a pocket of gas between the liquid free-surface and the tank wall. As the point of minimum separation between the liquid free-surface and the wall moves horizontally away from $x=0$, there is a concomitant bifurcation of the pressure profiles, with the maximum pressure subsequently obtained near the new point of minimum separation between the free surface and the wall. Figure 3 shows profiles at non-dimensional integer time steps, except for the final thicker solid profile which is shown just before touchdown. The thick dotted line is the profile at $t=0$, the instant touchdown would have occurred in the absence of cushioning, while the solid lines correspond to profiles at positive times, indicating gas cushioning delays the impact of the liquid with the wall.

As the value of $C$ increases, the pressure build-up in the gas film additionally induces condensation from the gas into the liquid. As gas is removed from the gas layer, the resulting pressure build-up is less (note the different pressure scales in figure 3), resulting in a reduced deceleration of the liquid free-surface and 
a smaller pocket of trapped gas. For (c) $C=4$, the free-surface curvature is negative throughout the preimpact cushioning and consequently a pocket of gas is not trapped, although the liquid is still decelerated prior to touchdown. In the experiments of [26], who use water and its vapour as a proxy for LNG in sloshing model tests, the overpressure generated during impact rapidly condenses the gas fraction, which is in good qualitative agreement with the theory presented herein. The trapping of a pocket of gas during the impact of a non-volatile gas is consistent with an oscillatory pressure signal in post-impact, driven by oscillations in the volume of the trapped gas pocket [2]. In impacts of volatile liquids, post-impact pressure oscillations are reduced or damped completely as the volume of gas trapped in impact is reduced [26, 41]. From equation (14), increasing values of $C$ correspond to increases in the characteristic normal impact velocity $V$, and, through the definition of $\varepsilon$, increases in the characteristic impact length scale $R$. Consequently, larger impacts at higher impact velocities are less likely to entrap gas, as in these cases, higher pressures induce more condensation. Therefore for wave impact with a vertical wall, as the impact size and velocity increases, the likelihood of mode (a) flip-through events (without gas entrainment), will increase, while mode (b) and mode (c) become less likely, as the gas necessary for flip-through in these modes will be condensed.

(a)

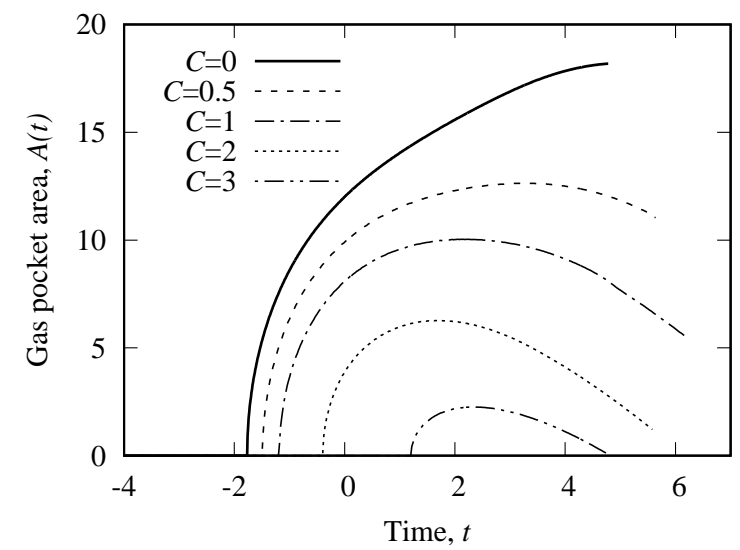

(b)

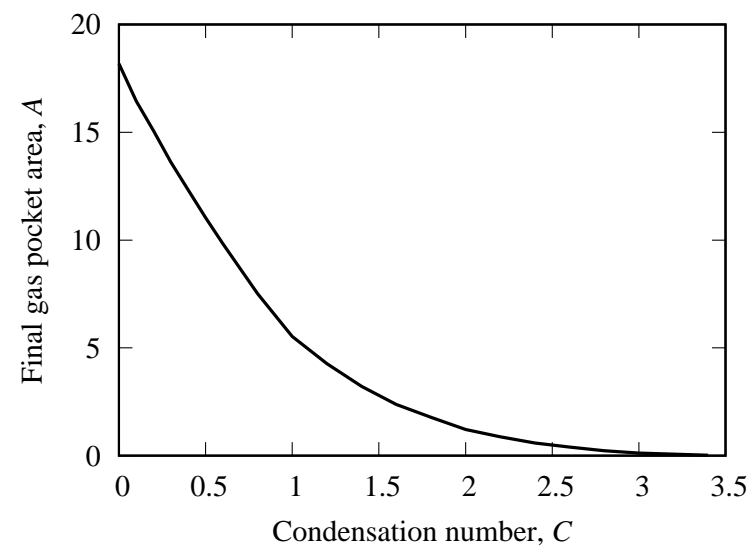

Figure 4: (a) The evolution of the area of gas trapped between the liquid free-surface and the wall and (b) the final area of the trapped gas pocket as a function of $C$.

To assess how changes in the value of $C$ effect the area of gas pocket enclosed by the solid body, the liquid free-surface and the horizontal locations of the points of minimum separation between the liquid free-surface and the wall $\pm x_{p}$, we calculate

$$
A(t)=\int_{-x_{p}(t)}^{x_{p}(t)}(s(x)-f(x, t)) \mathrm{d} x
$$

Gas is trapped in this region as the pressure gradient is zero at $\pm x_{p}$ and consequently, while the position of the local pressure maximum can evolve over time, the horizontal gas velocity equation (17) implies there is no net gas flow relative to this point. Figure 4(a) shows the evolution of $A(t)$ for a range of values of $C$, 
with the trapped area only increasing from zero once the liquid free-surface profile bifurcates. As expected the area of the trapped gas pocket decreases, with increasing values of $C$. For $C>0$, the gas pocket area initially increases after the bifurcation of the liquid free-surface, before the area subsequently decreases due to condensation induced by the pressure build-up. The final gas pocket area at touchdown $A_{p}$, as a function of $C$, is shown in figure 4(b). This indicates that a gas pocket is not trapped for $C \gtrsim 3.4$. From the definition of $C$ in equation (14), this gives a criterion on the formation of a trapped gas pocket, with a trapped gas pocket not being predicted for impact velocities

$$
V_{\text {crit }} \gtrsim \frac{3.25 \pi^{3 / 8} \mu_{g}^{1 / 4} \tilde{p}_{e}(\tilde{T})^{3 / 4}}{\rho_{l} R^{1 / 4} \mathcal{R}_{g}^{3 / 8} \tilde{T}^{3 / 8}} .
$$

Further experimental investigation is necessary to determine the practical value of this criterion, which is illustrated graphically in figure 5 . However, for a wide range of length scales appropriate for sloshing impacts, gas entrapment is not predicted for characteristic impact velocities greater than $0.05 \mathrm{~m} \mathrm{~s}^{-1}$ (indicated by the horizontal dashed line in figure 5). The theory developed herein requires that the gas pressure $p_{g} \rightarrow 0$ as $|x| \rightarrow 0$, and consequently it is not possible to maintain a net gas pressure gradient and a net gas mass flux across the cushioning region. Wave impacts with vertical walls (figure 1(c)), may involve a net gas flux in the cushioning region as an increase in gas pressure drives gas out of the developing cavity, and hence the extension of the model and equation (25) to incorporate such a flux is of potential future interest.

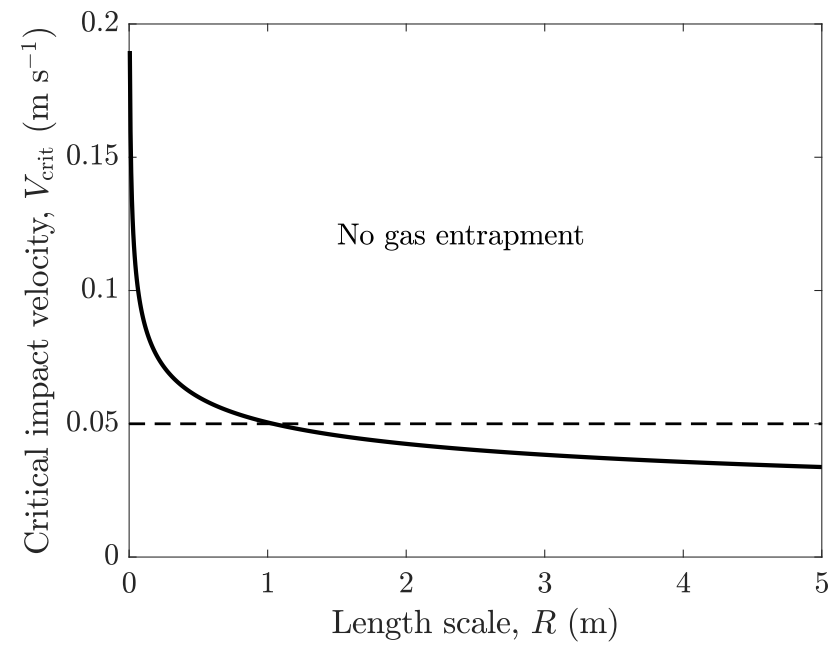

Figure 5: The critical normal impact velocity required to eliminate gas entrainment as a function of the characteristic impact length scale.

In cases where a gas pocket exists, its horizontal extent in dimensional variables is determined by the 
horizontal length scale, with touchdown expected at

$$
\tilde{x}_{p}=\frac{\mu_{g}^{1 / 3} R^{2 / 3}}{\rho_{l}^{1 / 3} V^{1 / 3}} x_{p}(C),
$$

for a dimensionless pre-factor $x_{p}(C)$. This pre-factor takes a value of approximately 4 in the non-volatile case, and decreases with increasing values of $C$. For a fixed value of $C$, equation (26) predicts that the area of the trapped gas pocket will increase with the initial radii of curvature of the liquid free-surface, as this increases the length of the free surface which can interact with the wall through gas cushioning, and decreases with an increase in the normal impact velocity, as there is less time for the liquid free-surface to deform before impact. In non-volatile three-dimensional liquid-solid impacts, this scaling law has been experimentally verified across a range of velocity and length scales, ranging from solid bodies with radius of curvature of $0.5 \mathrm{~m}$ hitting an initially quiescent liquid [18], to millimetre sized droplets [22]. Table 2 contains comparable scaling laws for the gas pocket volume $\tilde{A}_{p}$, characteristic gas layer height $\tilde{h}_{p}$, impact time delay $\tilde{t}_{p}$, and the characteristic pressure $\tilde{p}_{p}$. These relationships show that for a given value of $C$, the area of the gas pocket, its characteristic vertical height and the time delay prior to impact all increase with $R$ and decrease with $V$, following the behaviour seen for the horizontal extent of the gas pocket. The characteristic impact pressure increases with both $R$ and $V$, as larger, more violent impacts carry more momentum.

Table 2: The dependence on model variables on the characteristic radii of curvature of the liquid free-surface $R$, and the normal impact velocity $V$.

\begin{tabular}{|l|l|}
\hline Variable & Dimensional dependence \\
\hline Gas pocket area & $\tilde{A}_{p}=\frac{\mu_{g} R}{\rho_{l} V} A_{p}(C)$ \\
Characteristic gas layer height & $\tilde{h}_{p}=\frac{\tilde{A}_{p}}{\tilde{x}_{p}}=\frac{\mu_{g}^{2 / 3} R^{1 / 3}}{\rho_{l}^{2 / 3} V^{2 / 3}} h_{p}(C)$ \\
Characteristic impact delay & $\tilde{t}_{p}=\frac{\mu_{g}^{2 / 3} R^{1 / 3}}{\rho_{l}^{2 / 3} V^{5 / 3}} t_{p}(C)$ \\
Characteristic pressure & $\tilde{p}_{p}=\frac{\rho_{l}^{4 / 3} V^{7 / 3} R^{1 / 3}}{\mu_{g}^{1 / 3}} p_{p}(C)$ \\
\hline
\end{tabular}

The time evolution of the minimum separation between the liquid free-surface and the wall (left), and the maximum pressure (right), are shown in figure 6 for a range of values of $C$. The thick dash line in figure 6(a) indicates the evolution of the minimum separation in the absence of cushioning, i.e. when the free surface maintains its initial shape $f(x, t)=-\frac{1}{2} x^{2}-t$, right up to the instant of first impact. Compared to this asymptote, gas cushioning delays the instant of touchdown, with the largest delays associated with the smallest values of $C$. With larger values of $C$, touchdown is less delayed, as more phase change occurs and so 
(a)

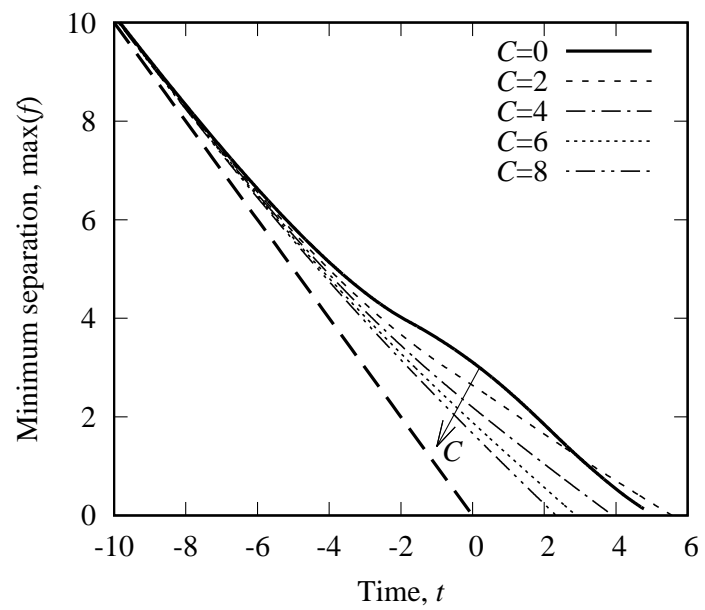

(b)

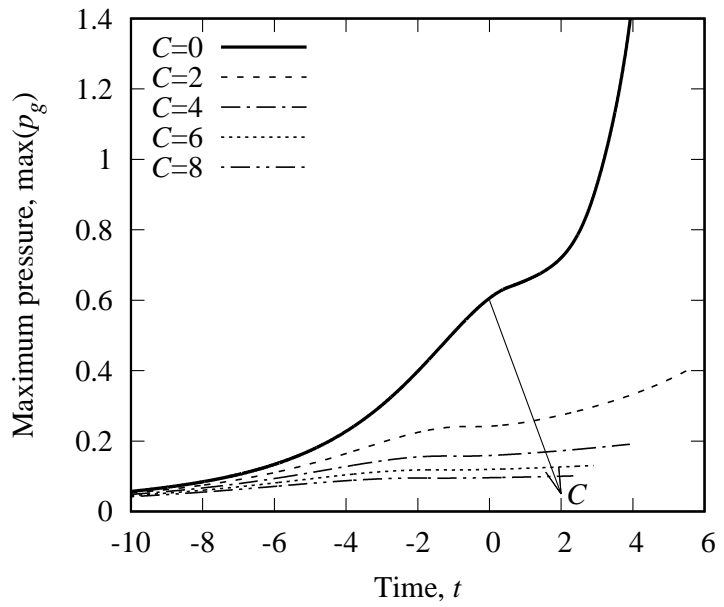

Figure 6: The evolution of (a) the minimum separation between the wall and the liquid free-surface, and (b) the maximum pressure as a function of time for a range of values of $C$. The straight thick dashed line in (a) indicates the expected evolution of the minimum free-surface separation in the absence of gas cushioning.

the pressure build-up and the free-surface deceleration are less. The higher pressures associated with $C=0$ are confirmed in figure 6(b), which shows that at any time during the cushioning, the maximum pressure decays monotonically as the value of $C$ increases.

Profiles of the mass flux due to condensation (top), the horizontal mass flux in the gas (middle), and the vertical velocity of the free surface (bottom), are shown in figure 7 for (a) $C=0$, (b) $C=2$ and (c) $C=4$. For figure 7(a), there is no mass flux across the interface as $C=0$. As $C$ increases the interfacial mass flux increases due to the pressure build-up in the gas layer driving condensation. For $C=4$, it is noticeable that the profiles for the condensation mass flux and pressure (see figure 3(c, bottom)), have bifurcated even when the corresponding free-surface profile retains a single global maximum throughout the pre-impact cushioning.

The horizontal mass flux along the gas layer is given by

$$
\dot{m}_{g}=\int_{f}^{s} \rho_{g} u_{g} \mathrm{~d} y=-\frac{\rho_{g}(s-f)^{3}}{12} \frac{\partial p_{g}}{\partial x} .
$$

As $C$ increases, there is a reduction in the variation of the mass flux between 0 and $x_{p}$, with very little flow observed in this region for $C>0$, compared to the non-volatile $C=0$ case. For $x>x_{p}$ there is a horizontal gas mass flux away from the point of impact as the gas is moved out of the path of the oncoming LNG. Additionally, there is less variability in the free-surface velocity for $C>0$, which is consistent with the earlier observations of reductions in the pressures and the area of the trapped gas pocket. For $x<x_{p}$, the gas pressure build-up locally decelerates the liquid free-surface, while for $x>x_{p}$ the liquid free-surface is locally accelerated towards touchdown. However, as the portion of the free surface with $x>x_{p}$, is initially further away from the wall than the free surface at $x=0$, the overall effect of gas cushioning is to delay impact. 
(a) $C=0$
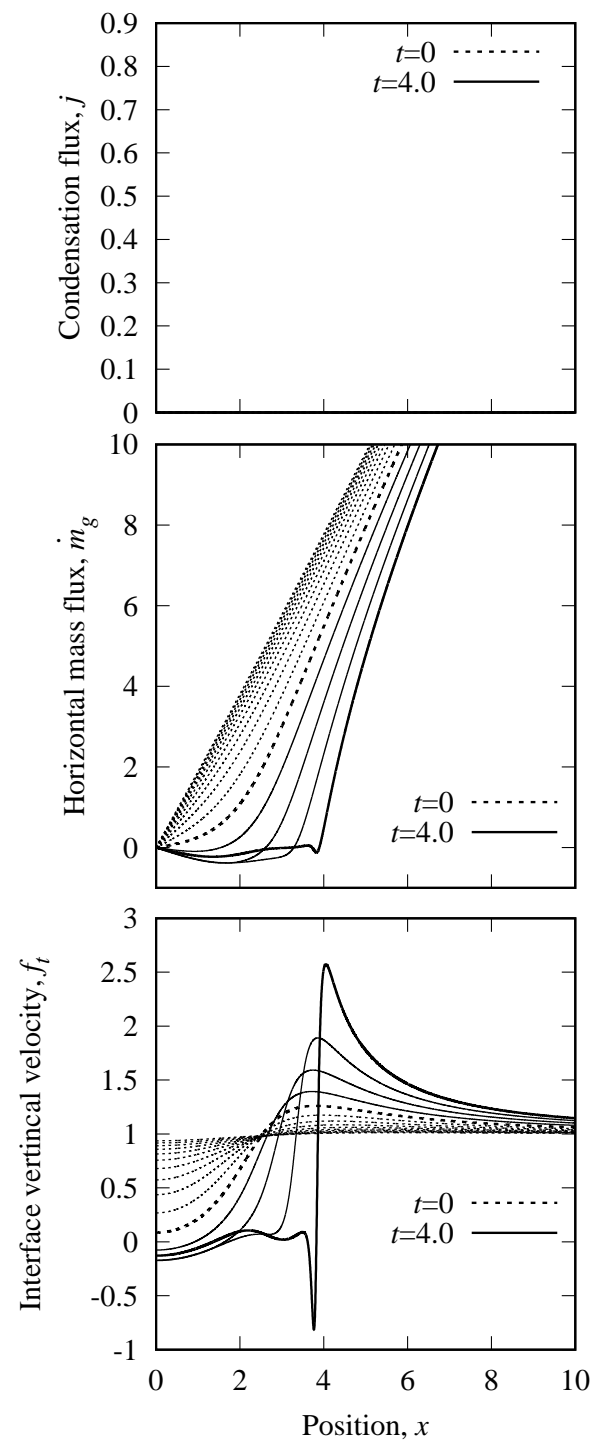

(b) $C=2$
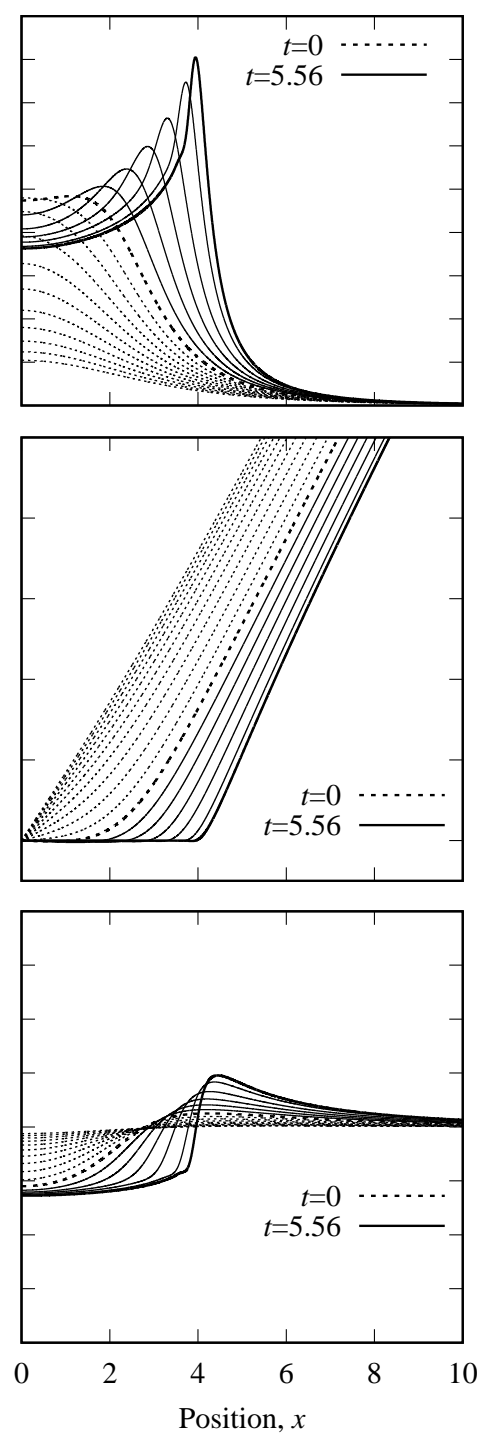

(c) $C=4$
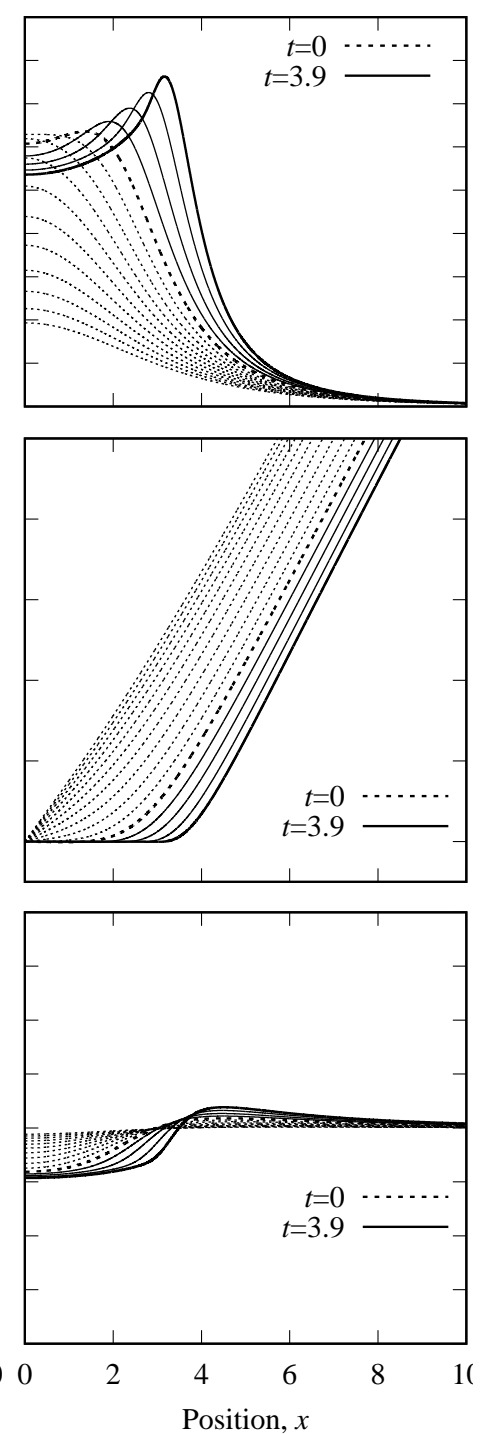

Figure 7: As figure 3, except showing profiles of the condensation flux (top), the horizontal mass flux in the gas film (middle), and the free-surface velocity (bottom).

The maximum free-surface velocities occur close to the point of initial touchdown. For a non-volatile liquid, the maximum velocity of the free surface towards the wall may exceed the bulk velocity by a factor of 2.5 . While the volume of liquid moving with this higher velocity is small compared to the bulk, it may be a factor in the determination of the maximum local impact pressure. As $C$ increases, both the decelerations and accelerations of the free surface decrease, as the gas pressure build-up induces interfacial mass transfer rather than decelerating the oncoming liquid. 


\subsection{Variation in compressibility parameter $\kappa$}

(a) $\kappa=0$
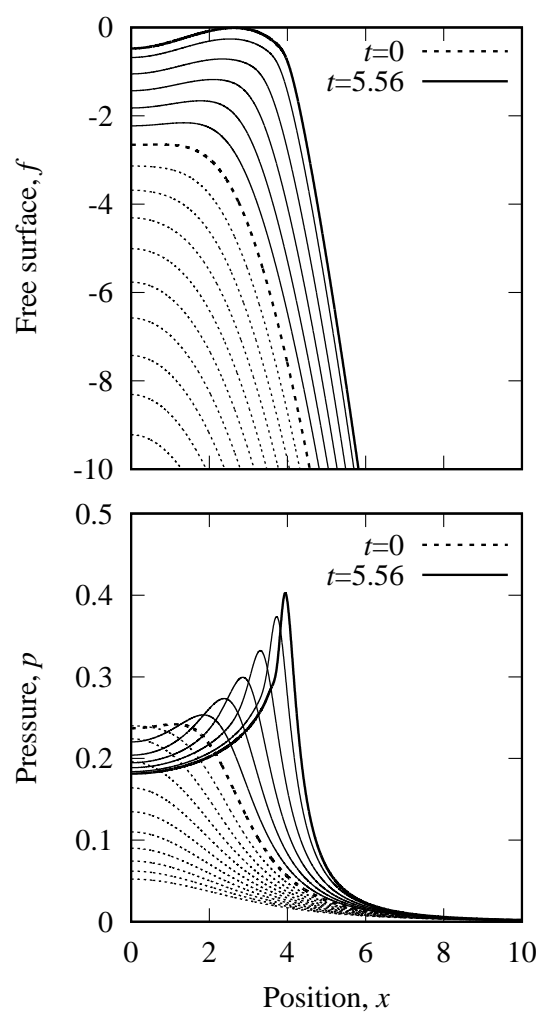

(b) $\kappa=2$
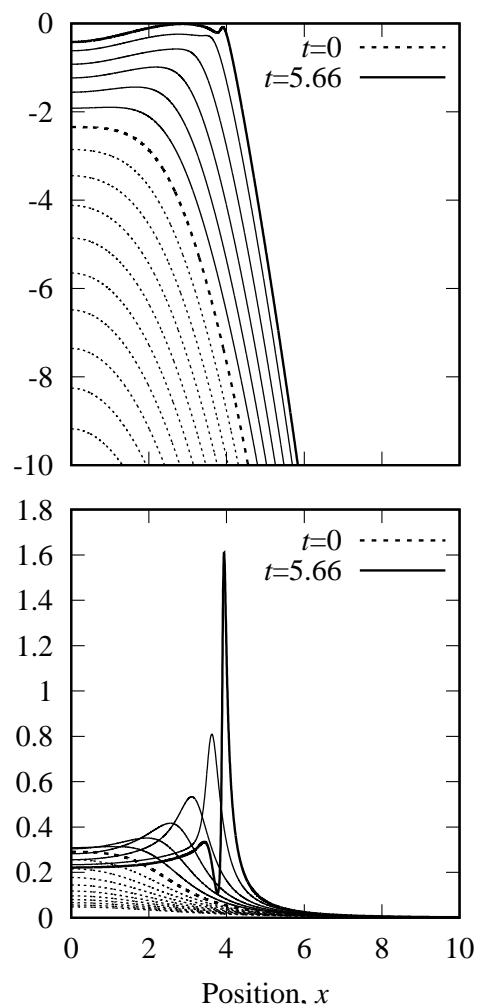

(c) $\kappa=8$
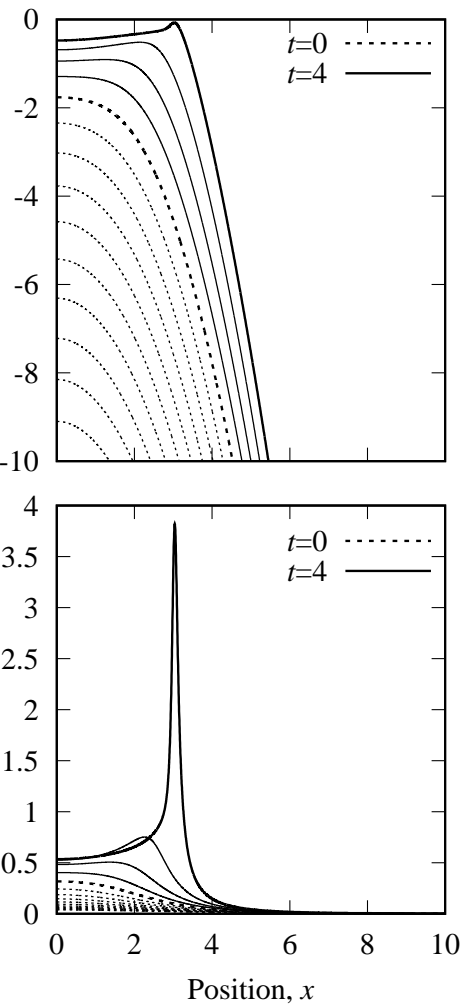

Figure 8: Profiles of the free surface (top) and pressure (bottom) for $C=2$ and $s(x)=0$, with (a) $\kappa=0$, (b) $\kappa=2$ and (c) $\kappa=8$. Profiles are shown at integer non-dimensional time increments, except for the final profile, which is shown just before touchdown. Profiles are symmetric about the vertical axis, with the solution domain matching the grey shaded regions of figure 1. Note that the pressure profiles are shown with different vertical scales to illustrate their features.

The effect of gas compressibility on an LNG-solid impact with $C=2, s(x)=0$ and (a) $\kappa=0$, (b) $\kappa=2$ and (c) $\kappa=8$ is illustrated in figure 8 . By considering the relative position of the free-surface profile at $t=0$ (the instant of touchdown in the absence of gas cushioning), it is evident that increasing values of $\kappa$ result in less deceleration of the liquid free-surface. The smaller separations between the liquid free-surface and the wall result in higher pressures as $\kappa$ increases (note the differences in the pressure scales in figure 8). There is some evidence of multiple touchdown points in the $\kappa=2$ case due to local variations in the pressure profiles just prior to touchdown.

The effect of the fluid density ratio on LNG impacts was investigated by Yung et al. [40], who conducted experimental sloshing tests for both non-volatile and volatile liquids. Boiling water was used as a proxy for LNG in volatile impacts, while a range of fluid density ratios where studied for non-volatile sloshing impacts by surrounding the liquid by gases of varying density. In the current framework, increasing the gas density, 
while the liquid density and other fluid properties remain constant has the effect of decreasing the value of $C$, while the value of $\kappa$ is unchanged. Consequently, a denser gas has no impact on the compressibility of the gas, but it does condense less readily.

\subsection{Impacts in alternative geometries}

(a) $\alpha=0$
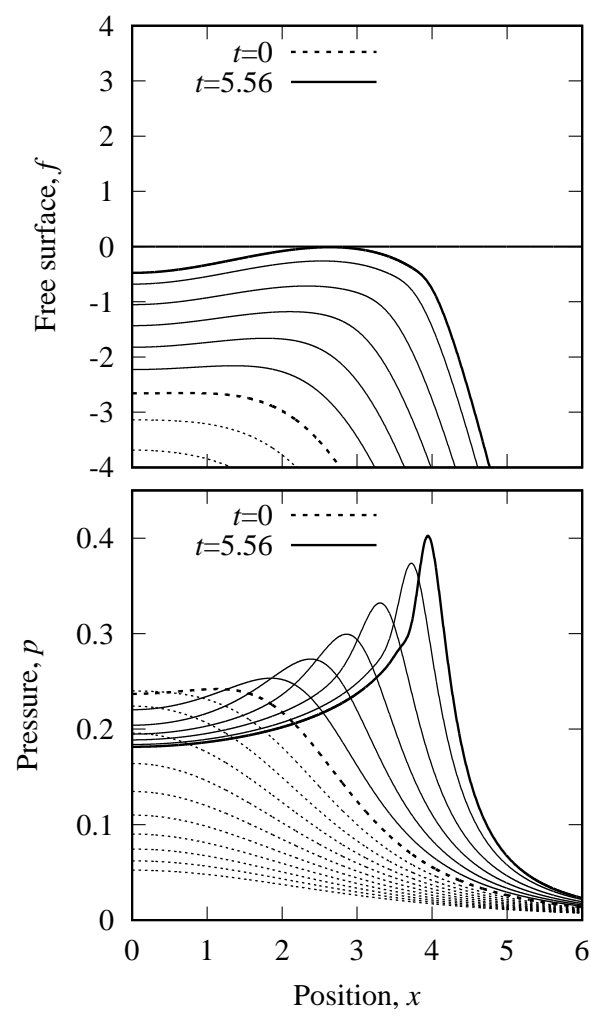

(b) $\alpha=1$
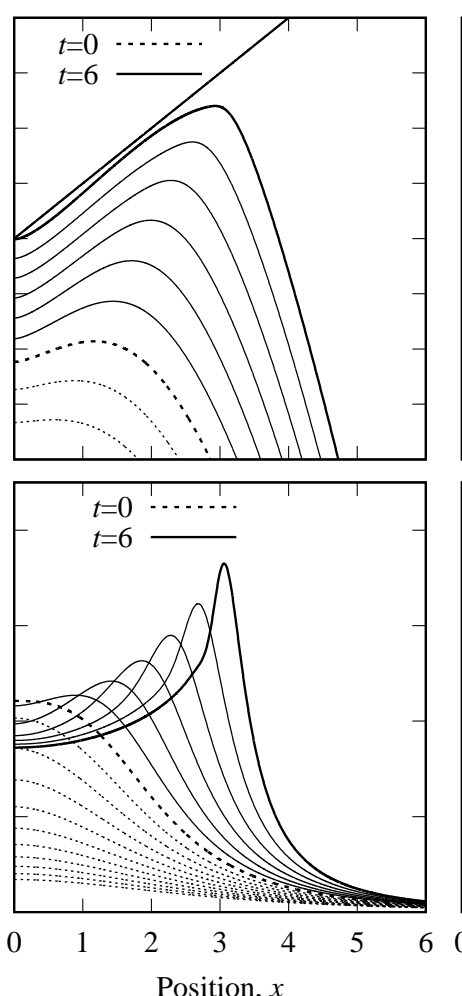

(c) $\alpha=2$
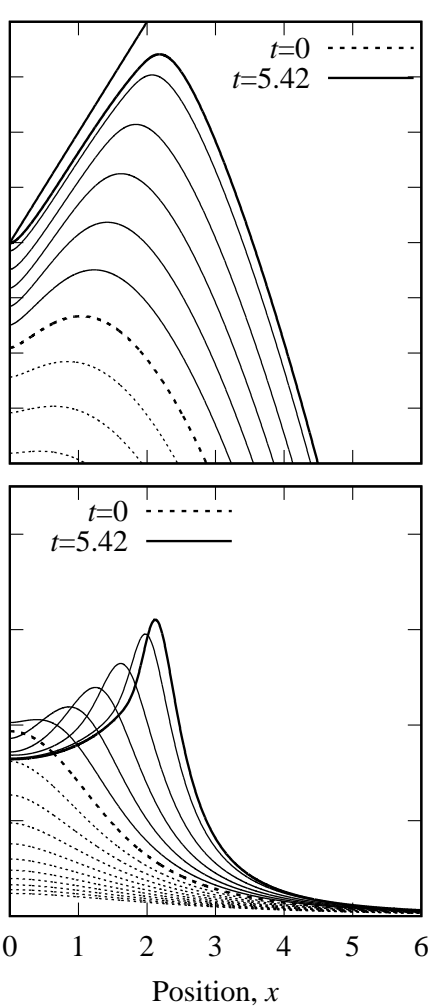

Figure 9: Profiles of the free surface (top) and pressure (bottom) for $C=2, \kappa=0$ and $s(x)=\alpha|x|$, with (a) $\alpha=0$, (b) $\alpha=1$ and (c) $\alpha=2$. Profiles are shown at integer non-dimensional time increments, except for the final profile, which is shown just before touchdown. The solution domain matches the grey shaded regions of figure 1 (b).

To investigate LNG-solid impacts comparable to a tip of an LNG jet running up a tank wall towards the corner of a tank, the shape of the wall is varied by taking $s(x)=\alpha|x|$. The symmetry of $s(x)$ about $x=0$ guarantees no-penetration of fluid through a vertical wall, which is assumed to exist at $x=0$. The liquid is still inviscid to leading order, while the influence on the gas of a no-slip boundary condition due to the vertical wall at $x=0$ is assumed to be negligible. In this configuration, the flow is equivalent to that induced by an impact with a symmetrical wedge with shape $s(x)=\alpha|x|$. The case $\alpha=0$ corresponds to impact with a roof at $90^{\circ}$ to the vertical wall, while $\alpha>0$ increases the internal angle the roof makes with the wall. Note that the lubrication approximation in the gas is retained, and so a non-dimensional gradient of the 
tank roof $\alpha=\mathcal{O}(1)$, corresponds to an $\mathcal{O}(\varepsilon)$ dimensional gradient. However, with this restriction, the effect of small increases in the angle the tank roof makes with the tank wall are investigated.

Figure 9 shows the impact of liquid with the corner of the tank for $C=2, \kappa=0$ and (a) $\alpha=0$, (b) $\alpha=1$ and (c) $\alpha=2$. Increasing the value of $\alpha$ reduces the horizontal extent of the gas cushioning region, as the angled roof reduces the length that lies within the interaction region for viscous cushioning. Consequently, the horizontal extent and volume of the trapped gas pocket falls with increasing values of $\alpha$. At each time step, the maximum pressure also falls, as larger values of $\alpha$ increase the separation between the liquid and the tank roof.

\section{Conclusions}

A model of LNG-solid pre-impact cushioning with volatile fluid phases is presented within the viscous gas and inviscid liquid distinguished limit of Smith et al. [35]. Due to cushioning induced local pressure increases above the local saturation pressure, condensation from the gas to the LNG is predicted. Compared to an equivalent impact involving a non-volatile liquid, condensation acts to lower pre-impact pressures and reduce the effectiveness of impact cushioning by the gas. Consequently, the liquid is decelerated less prior to impact and therefore may result in higher post-impact pressures as less momentum is dissipated before impact. Flip-through impact modes, which required entrained gas, are also less likely when condensation is present, as the gas will be condensed prior to impact. The model is limited to the pre-touchdown cushioning stage of a liquid-solid impact and consequently is unable to predict the oscillatory pressure signals, which are observed after initial touchdown $[26,41]$. However, the model is a useful predictive tool in determining the volume of gas trapped during cushioning. Scaling laws are proposed for the extent of any trapped gas pocket as a function of the impact parameters. A critical characteristic normal impact velocity is determined, and a pocket of trapped gas is not predicted at impact velocities greater than this. Extensions to the basic model illustrate how gas compressibility can be incorporated and how impacts in more complicated geometries can be simulated.

The use of the viscous gas and inviscid liquid distinguished limit of Smith et al. [35] bounds the applicability of the model to small and moderate LNG sloshing impacts. However, for impacts involving water, the full range of characteristic impact sizes seen in the scale model tests of [26] can be investigated. This is because inertia in the gas cushion is shown to be significant at leading order in larger-scale LNG sloshing impacts, while this term is less important in impacts involving water. As future work, gas inertia may be incorporated within the current modelling framework to investigate larger scale volatile impact cushioning, either by incorporating moderate inertia to an otherwise viscous thin fluid film following the approach of [34] or by adding phase change to the inviscid gas and inviscid liquid impact cushioning model of Wilson [37]. 


\section{References}

[1] Abrahamsen, B. C., \& Faltinsen, O. M. (2009). Decay of air cavity slamming pressure oscillations during sloshing at high fillings. In Proceedings of 24th International Workshop on Water Waves and Floating Bodies.

[2] Abrahamsen, B. C., \& Faltinsen, O. M. (2011). The effect of air leakage and heat exchange on the decay of entrapped air pocket slamming oscillations. Phys. Fluids, 23, 102107. doi:10.1063/1.3638612.

[3] Abrahamsen, B. C., \& Faltinsen, O. M. (2012). The natural frequency of the pressure oscillations inside a water-wave entrapped air pocket on a rigid wall. J. Fluid. Struct., 35, 200-212. doi:10.1016/j. jfluidstructs.2012.07.004.

[4] Abrahamsen, B. C., \& Faltinsen, O. M. (2013). Scaling of entrapped gas pocket slamming events at dissimilar Euler number. J. Fluid. Struct., 40, 246-256. doi:10.1016/j.jfluidstructs.2013.04.009.

[5] Ancellin, M., Brosset, L., \& Ghidaglia, J.-M. (2012). Influence of phase transition on sloshing impact pressures described by a generalized Bagnold's model. In Proceedings of the 22nd International Offshore and Polar Engineering Conference, 17-22 June, Rhodes, Greece (pp. 300-310). International Society of Offshore and Polar Engineers.

[6] Arswendy, A., \& Moan, T. (2015). Strength and stiffness assessment of an LNG containment system Crushing and buckling failure analysis of plywood components. Eng. Fail. Anal., 48, 247-258. doi:10. 1016/j . engfailanal.2014.11.017.

[7] Bagnold, R. A. (1939). Interim report on wave pressure research. J. Inst. Civil Eng., 12, 201-226. doi:10.1680/ijoti.1939.14539.

[8] Braeunig, J.-P., Brosset, L., Dias, F., \& Ghidaglia, J.-M. (2010). On the effect of phase transition on impact pressures due to sloshing. In The Proceedings of The Twentieth International Offshore and Polar Engineering Conference, 20-25 June, Beijing, China ISOPE-I-10-182 (pp. 53-61). International Society of Offshore and Polar Engineers.

[9] Burelbach, J. P., Bankoff, S. G., \& Davis, S. H. (1988). Nonlinear stability of evaporating/condensing liquid films. J. Fluid Mech., 195, 463-494. doi:10.1017/S0022112088002484.

[10] Colagrossi, A., Lugni, C., \& Brocchini, M. (2010). A study of violent sloshing wave impacts using an improved SPH method. J. Hydraul. Res., 48, 94-104. doi:10.1080/00221686.2010.9641250.

[11] Delorme, L., Colagrossi, A., Souto-Iglesias, A., Zamora-Rodríguez, R., \& Botía-Vera, E. (2009). A set of canonical problems in sloshing, Part I: Pressure field in forced roll-comparison between experimental results and SPH. Ocean Eng., 36, 168-178. doi:10.1016/j .oceaneng.2008.09.014. 
[12] Dias, F., Ghidaglia, J.-M., \& Le Coq, G. (2007). On the fluid dynamics models for sloshing. In The Proceedings of The Seventeenth International Offshore and Polar Engineering Conference, 1-6 July, Lisbon, Portugal (pp. 88-97). International Society of Offshore and Polar Engineers.

[13] Duchemin, L., \& Josserand, C. (2011). Curvature singularity and film-skating during drop impact. Phys. Fluids, 23, 091701. doi:10.1063/1.3640028.

[14] Duchemin, L., \& Josserand, C. (2012). Rarefied gas correction for the bubble entrapment singularity in drop impacts. C. R. Mec., 340, 797-803. doi:10.1016/j.crme.2012.10.028.

[15] Faltinsen, O. M., \& Timokha, A. N. (2009). Sloshing. Cambridge University Press, Cambridge.

[16] Gavory, T., \& de Seze, P. E. (2009). Sloshing in membrane LNG carriers and its consequences from a designer's perspective. In Proceedings of the Nineteenth International Offshore and Polar Engineering Conference, Osaka, Japan, June 21-26 ISOPE-I-09-041 (pp. 13-20). International Society of Offshore and Polar Engineers.

[17] Gong, K., Shao, S., Liu, H., Wang, B., \& Tan, S.-K. (2016). Two-phase SPH simulation of fluid-structure interactions. J. Fluid. Struct., 65, 155-179. doi:10.1016/j.jfluidstructs.2016.05.012.

[18] Hicks, P. D., Ermanyuk, E. V., Gavrilov, N. V., \& Purvis, R. (2012). Air trapping at impact of a rigid sphere onto a liquid. J. Fluid Mech., 695, 310-320. doi:10.1017/jfm.2012.20.

[19] Hicks, P. D., \& Purvis, R. (2010). Air cushioning and bubble entrapment in three-dimensional droplet impacts. J. Fluid Mech., 649, 135-163. doi:10.1017/S0022112009994009.

[20] Hicks, P. D., \& Purvis, R. (2013). Liquid-solid impacts with compressible gas cushioning. J. Fluid Mech., 735, 120-149. doi:10.1017/jfm.2013.487.

[21] Hicks, P. D., \& Purvis, R. (2017). Gas-cushioned droplet impacts with a thin layer of porous media. J. Eng. Math., 102, 65-87. doi:10.1007/s10665-015-9821-y.

[22] Li, E. Q., \& Thoroddsen, S. T. (2015). Time-resolved imaging of a compressible air disc under a drop impacting on a solid surface. J. Fluid Mech., 780,636-648. doi:10.1017/jfm.2015.466.

[23] Lugni, C., Brocchini, M., \& Faltinsen, O. M. (2006). Wave impact loads: The role of the flip-through. Phys. Fluids, 18, 122101. doi:10.1063/1.2399077.

[24] Lugni, C., Brocchini, M., \& Faltinsen, O. M. (2010). Evolution of the air cavity during a depressurized wave impact. II. The dynamic field. Phys. Fluids, 22, 056102. doi:10.1063/1.3409491. 
[25] Lugni, C., Miozzi, M., Brocchini, M., \& Faltinsen, O. M. (2010). Evolution of the air cavity during a depressurized wave impact. I. The kinematic flow field. Phys. Fluids, 22, 056101. doi:10.1063/1. 3407664.

[26] Maillard, S., \& Brosset, L. (2009). Influence of density ratio between liquid and gas on sloshing model test results. In In Proceedings of The Nineteenth International Offshore and Polar Engineering Conference, 21-26 June, Osaka, Japan ISOPE-I-09-043 (pp. 167-174). International Society of Offshore and Polar Engineers.

[27] Mandre, S., Mani, M., \& Brenner, M. P. (2009). Precursors to splashing of liquid droplets on a solid surface. Phys. Rev. Lett., 102, 134502. doi:10.1103/PhysRevLett.102.134502.

[28] Oron, A., Davis, S. H., \& Bankoff, S. G. (1997). Long-scale evolution of thin liquid films. Rev. Mod. Phys., 69, 931-980. doi:10.1103/RevModPhys.69.931.

[29] Palmer, H. J. (1976). The hydrodynamic stability of rapidly evaporating liquids at reduced pressure. J. Fluid Mech., 75, 487-511. doi:10.1017/s0022112076000347.

[30] Peregrine, D. H. (2003). Water wave impact on walls. Annu. Rev. Fluid Mech., 35, 23-43. doi:10.1146/ annurev.fluid.35.101101.161153.

[31] Persad, A. H., \& Ward, C. A. (2016). Expressions for the evaporation and condensation coefficients in the Hertz-Knudsen relation. Chem. Rev., 116, 7727-7767. doi:10.1021/acs.chemrev.5b00511.

[32] Purvis, R., \& Smith, F. T. (2004). Air-water interactions near droplet impact. Eur. J. Appl. Math., 15, 853-871. doi:10.1017/S0956792504005674.

[33] Quéré, D. (2013). Leidenfrost dynamics. Annu. Rev. Fluid Mech., 45, 197-215. doi:10.1146/annurev-fluid-011212-140709.

[34] Sivapuratharasu, M., Hibberd, S., Hubbard, M. E., \& Power, H. (2016). Inertial effects on thin-film wave structures with imposed surface shear on an inclined plane. Physica D, 325, 86-97. doi:10.1016/ j.physd.2016.03.008.

[35] Smith, F. T., Li, L., \& Wu, G. X. (2003). Air cushioning with a lubrication/inviscid balance. J. Fluid Mech., 482, 291-318. doi:10.1017/S0022112003004063.

[36] Topliss, M. E., Cooker, M. J., \& Peregrine, D. H. (1992). Pressure ocsillations during wave impact on vertical walls. In Proceedings of the 23rd Conference on Coastal Engineering, Venice, Italy, 1992. (pp. 1639-1650). volume 23. 
[37] Wilson, S. K. (1991). A mathematical model for the initial stages of fluid impact in the presence of a cushioning fluid layer. J. Eng. Math., 25, 265-285. doi:10.1007/BF00044334.

[38] Wilson, S. K., Davis, S. H., \& Bankoff, S. G. (1999). The unsteady expansion and contraction of a long two-dimensional vapour bubble between superheated or subcooled parallel plates. J. Fluid Mech., 391, 1-27. doi:10.1017/S0022112099004516.

[39] Yan, R., Monaghan, J., Valizadeh, A., \& Xu, F. (2015). The effect of air on solid body impact with water in two dimensions. J. Fluid. Struct., 59, 146-164. doi:10.1016/j.jfluidstructs.2015.08.015.

[40] Yung, T.-W., Ding, J., He, H., \& Sandström, R. (2009). LNG sloshing: characteristics and scaling laws. Int. J. Offshore Polar Eng., 19, 264-270.

[41] Yung, T.-W., Minta, M., He, H., \& Gentry, M. (2010). Dynamics of vapor pockets at phase boundary: an application in LNG sloshing. In Proceedings of the 29th International Conference on Ocean, Offshore and Arctic Engineering: Volume 1, Shanghai, China, June 6-11 OMAE2010-21191 (pp. 689-697). doi:10.1115/OMAE2010-21191. 